\title{
Restoration of the cAMP Second Messenger Pathway Enhances Cardiac Preservation for Transplantation in a Heterotopic Rat Model
}

D. Pinsky, M. Oz, H. Liao, S. Morris, J. Brett, R. Sciacca, M. Karakurum, M. Van Lookeren Campagne, J. Platt, * R. Nowygrod, S. Koga, and D. Stern

Departments of Physiology, Medicine, and Surgery, Columbia University, College of Physicians and Surgeons, New York 10032; and *Duke University School of Medicine, Department of Surgery, Durham, North Carolina 27710

\begin{abstract}
Current organ preservation strategies subject graft vasculature to severe hypoxia ( $\mathrm{PO}_{2} \sim 20$ Torr $)$, potentially compromising vascular function and limiting successful transplantation. Previous work has shown that CAMP modulates endothelial cell (EC) antithrombogenicity, barrier function, and leukocyte / EC interactions, and that hypoxia suppresses EC cAMP levels. To explore the possible benefits of cAMP analogs / agonists in organ preservation, we used a rat heterotopic cardiac transplant model; dibutyryl cAMP added to preservation solutions was associated with a time- and dose-dependent increase in the duration of cold storage associated with successful graft function. Preservation was also enhanced by 8 -bromo-cAMP, the $S_{p}$ isomer of adenosine $3^{\prime}, 5^{\prime}$ monophosphorothioate, and types III (indolidan) and IV (rolipram) phosphodiesterase inhibitors. Neither butyrate alone nor 8-bromoadenosine were effective, and the cAMP-dependent protein kinase antagonist $R_{p}$ isomer of adenosine 3 ',5'monophosphorothioate prevented preservation enhancement induced by 8-bromo-cAMP. Grafts stored with dibutyryl cAMP demonstrated a 5.5-fold increase in blood flow and a 3.2-fold decreased neutrophil infiltration after transplantation. To explore the role of cAMP in another cell type critical for vascular homeostasis, vascular smooth muscle cells were subjected to hypoxia, causing a time-dependent decline in cAMP levels. Although adenylate cyclase activity was unchanged, diminished oxygen tensions were associated with enhanced phosphodiesterase activity ( 59 and $30 \%$ increase in soluble types III and IV activity, respectively). These data suggest that hypoxia or graft ischemia disrupt vascular homeostasis, at least in part, by perturbing the cAMP second messenger pathway. Supplementation of this pathway provides a new approach for enhancing cardiac preservation, promoting myocardial function, and maintaining vascular homeostatic properties. ( $J$. Clin. Invest. 1993. 92:2994-3002.) Key words: organ preservation • transplantation • cAMP • hypoxia $\bullet$ blood vessels
\end{abstract}

\section{Introduction}

Preservation of hearts after explantation is a major limitation in clinical heart transplantation. Current approaches to cardiac

Address correspondence to Dr. David Pinsky/Dr. David Stern, Department of Physiology, P\&S 11-518, Columbia University College of Physicians and Surgeons, 630 West 168th Street, New York, NY 10032. 1993.

Received for publication 11 March 1993 and in revised form $21 \mathrm{July}$

J. Clin. Invest.

(C) The American Society for Clinical Investigation, Inc. 0021-9738/93/12/2994/09 \$2.00

Volume 92, December 1993, 2994-3002 preservation include attention to electrolyte balance, and the addition of impermeant anions, colloids, antioxidants, agents to limit entry of calcium, and metabolic substrates to promote the generation of high energy phosphate compounds on reperfusion (1-15). In view of the importance of vascular homeostatic properties such as permeability/barrier function, thrombogenicity/antithrombogenicity, expression of leukocyte-adherence molecules, and vasomotor tone, another important consideration in the design of cardiac preservation strategies is the maintenance of vascular integrity and function. These vascular homeostatic properties are modulated by the major cellular components of the vessel wall, including endothelial and smooth muscle cells. Although endothelial cells (ECs) ${ }^{1}$ were once thought of as a passive, nonwettable conduit for blood flow, it has become clear that they play a dynamic role in the control of vascular properties (16-19). An important intracellular second messenger associated with $\mathrm{EC}$ regulation of vascular homeostatic properties is CAMP, which maintains EC function and prevents the induction of procoagulant activity in response to certain cytokines (20-24). When ECs are exposed to hypoxia, barrier function is diminished in parallel with a fall in levels of intracellular cAMP. This barrier function is almost completely normalized by the addition of cAMP analogues (25). Furthermore, ischemia-reperfusion induced increases in microvascular permeability is prevented by agents which elevate cAMP in a perfused lung model (26).

In view of the ischemia/hypoxia that accompanies harvest and storage of an explanted heart, we hypothesized that addition of CAMP analogues to preservation solutions would exert a beneficial effect on vascular function and organ preservation. The results of our studies indicate that agents which raise intracellular cAMP (such as rolipram or indolidan) or cAMP analogues/agonists (including $N^{6}, \mathrm{O}^{2}$-dibutyryl cAMP [dbcAMP], 8-bromoadenosine 3',5'monophosphate [8-Br-cAMP], and the $S_{p}$ isomer of adenosine $3^{\prime}, 5^{\prime}$ monophosphorothioate [ $S_{p}$-cAMPS]) promote cardiac preservation in a rat heterotopic transplant model. cAMP analogues appear to exert their effects via a pathway that involves, at least in part, stimulation of cAMP-dependent protein kinase, which results in enhanced blood flow and decreased leukocyte infiltration of the graft. To further explore the role of the vasculature in cAMP-induced enhancement of graft preservation, vascular smooth muscle cells (SMCs) were subjected to hypoxia to determine whether a

\footnotetext{
1. Abbreviations used in this paper: 8-Br-cAMP, 8-bromoadenosine 3',5'monophosphate; EC, endothelial cell; db-cAMP, $N^{6}, \mathrm{O}_{2}^{\prime}$-dibutyryl adenosine 3',5'monophosphate; LR, lactated Ringer's solution; PDE, phosphodiesterase; PKA, cyclic AMP-dependent protein kinase; $\mathbf{R}_{\mathbf{p}}$ cAMPS, $R_{p}$ isomer of adenosine $3^{\prime}, 5^{\prime}$ monophosphorothioate; $S_{p}-$ cAMPS, $S_{p}$ isomer of adenosine 3',5'monophosphorothioate; SMC, smooth muscle cell; UW, University of Wisconsin solution.
} 
similar decline in intracellular cAMP would occur in vascular SMCs exposed to hypoxia as has been reported for ECs (25). In this communication, we demonstrate that vascular SMCs exposed to hypoxia show a time-dependent decline in cAMP levels, associated with an increase in phosphodiesterase activity, notably types III and IV. These findings are important because SMCs, as the cellular end-regulators of blood flow, are critical to vascular homeostasis, and because cAMP is known to play an important role in the regulation of vasomotor tone (27). Taken together, these studies indicate that a new level of intervention, addition of cAMP analogues to preservation solutions for maintenance of vascular function, improves cardiac preservation after prolonged hypothermic storage.

\section{Methods}

\section{Heterotopic rat cardiac transplant model (28)}

Preparation of preservation solution. Preservation solutions included lactated Ringer's (LR) solution (Baxter Healthcare Corp., Edison, NJ), or LR supplemented with rolipram (ZK 62 711; Schering AG, Berlin, Germany), indolidan (LY1951 15; Lilly Research Laboratories, Indianapolis, IN), sodium butyrate, 8-bromoadenosine, 8-bromoadenosine 3',5'monophosphate (8-Br-cAMP) (Sigma Chemical Co., St. Louis, MO), the $R_{p}$-isomer of adenosine 3',5'monophosphorothioate ( $R_{p}$-cAMPS) (Biolog Life Science Institute, La Jolla, CA), $S_{p}$-cAMPS (Biolog Life Science Institute), and db-cAMP) (Aldrich Chemical Co., Milwaukee, WI). Components were combined within $4 \mathrm{~h}$ of donor cardiectomy, and preservation solutions were kept at $4^{\circ} \mathrm{C}$. University of Wisconsin solution (UW) (2-4) was purchased from Du Pont Pharmaceuticals (Wilmington, DE).

Donor cardiectomy. Male Sprague-Dawley rats (350-450 g, Camm Research, Wayne, NJ) were anesthetized, heparinized ( $1,000 \mathrm{U}$ of heparin given intravenously), and the donor heart was removed in the following manner: a midline incision was performed, followed by rapid cardioplegic arrest with $5 \mathrm{ml}$ of cold $\left(4^{\circ} \mathrm{C}\right)$ high potassium solution $(\mathrm{NaCl}, 0.9 \%$, and $\mathrm{KCl}, 40 \mathrm{mEq} /$ liter $)$. The heart was maintained at $4^{\circ} \mathrm{C}$ throughout the harvest procedure by the application of a cold saline soaked gauze over its surface. The inferior and superior vena cavae were ligated, the aortic and pulmonary artery roots were transected, the pulmonary veins were ligated, and the entire heart was excised and immersed in the cold preservation solution. The aortic root was flushed with $\sim 15 \mathrm{ml}$ of the cold preservation solution until the coronary arteries became visibly clear, and the heart remained in the cold storage solution for the indicated duration until the time of transplantation.

Heterotopic transplant. The same breed/size of rat was used and similarly anesthetized, but not given heparin. A midline abdominal incision was made, the infrarenal aorta and vena cava were occluded with a side-biting vascular clamp, and longitudinal incisions were made in each vessel. The donor heart was removed from the preservation solution and kept moist with saline-soaked gauze. The donor heart's ascending aorta was anastomosed to the recipient's abdominal aorta, and the donor's pulmonary artery was anastomosed to the recipient's inferior vena cava using 8-0 monofilament suture material. Blood flow was restored to the graft (by removal of the clamp) exactly $1 \mathrm{~h}$ after removal from the cold preservation solution. Pulsation of the donor aortic stump was observed to assure patency of the anastomosis. $10 \mathrm{~min}$ after removal of the cross-clamp, an electrocardiogram was taken (Grass Polygraph, Quincy, MA), and the heart was judged by the same blinded investigators throughout based on the presence/absence of regular contractions and a transplant score ( $0-5$, worst-best, respectively) using the following criteria: contraction (poor $=0$, average $=1$, vigorous $=2$ ), tissue turgor ( hard $=0$, average $=1$, soft $=2$ ), and color ( dusky $=0$, pink $=1)$. Overall, a graft was considered to have survived if regular contractions were observed with confirmation of corresponding depolarizations by electrocardiogram, with a transplant score $\geq 2$.
The heterotopic cardiac transplant experiments performed in these studies were done by a single surgeon after establishment of this model and acquisition of surgical expertise, with control and experimental groups interspersed throughout the study period to prevent a time-dependent bias pertaining to surgical skills.

Perfusion of donor graft. $10 \mathrm{~min}$ after restoration of blood flow, a suspension of latex microspheres $(0.5 \mathrm{ml}$ of $10-\mu \mathrm{m}$ diameter microspheres at a final concentration in $10^{6} / \mathrm{ml}$ in phosphate-buffered saline containing Tween $80,0.05 \%$ ) (E-Z Trac, Los Angeles, CA) was injected during $30 \mathrm{~s}$ into the donor aortic root. After $1 \mathrm{~min}$, the heart was rapidly excised, rinsed free of intracavitary and surface blood, and the atria and great vessels were removed. The ventricles were cut into pieces, and each piece was weighed, digested by alkaline hydrolysis, centrifuged, and washed according to the manufacturer's instructions. Beads in each piece were then counted in a hemocytometer (Improved Neubauer; VWR Scientific, Piscataway, NJ). Results are expressed as number of spheres per $(\times 40)$ field per gram of ventricle. A minimum of ten fields was counted and the mean \pm SEM was reported.

Myeloperoxidase assay/histology. $10 \mathrm{~min}$ after restoration of blood flow, transplanted hearts were excised and the aortic root was flushed for $5 \mathrm{~min}$ (rate of $2 \mathrm{ml} / \mathrm{min}$ controlled by roller pump) with normal saline (Baxter Healthcare Corp.), the atria and great vessels were removed, and intracavitary blood was evacuated. Remaining ventricular pieces were weighed and homogenized in phosphate buffer ( 50 $\mathrm{mM} ; \mathrm{pH} 7.4,5 \mathrm{ml} / \mathrm{gm}$ of tissue) containing $0.5 \%$ hexadecyltrimethylammonium bromide (Sigma Chemical Co.) and frozen at $-80^{\circ} \mathrm{C}$. The myeloperoxidase assay was performed, as described (29), by thawing the sample, centrifuging at $40,000 \mathrm{~g}$ for $10 \mathrm{~min}$ at $4^{\circ} \mathrm{C}$, and decanting the supernatant, which was assayed for myeloperoxidase activity using standard chromogenic spectrophotometric technique: test sample $(0.1$ $\mathrm{ml}$ ) was added to phosphate buffer $(50 \mathrm{mM}, \mathrm{pH} 6.0$ ) containing $O$ dianisidine dihydrochloride (Sigma Chemical Co.), hydrogen peroxide $(0.0005 \%)$, and change in absorbance at $460 \mathrm{~nm}$ was measured during 5 min (increase in OD was linear during this time interval). For histologic study, cardiac graft samples were fixed in an ascending series of aldehydes, embedded, sectioned, and stained with hematoxylin and eosin.

Vascular smooth muscle cell culture, assays for cAMP, phosphodiesterase, and adenylate cyclase. Primary vascular smooth muscle cell cultures were obtained from bovine adrenal vessels by collagenase digestion and a series of differential adhesion steps. Cultures were characterized morphologically (30) and by the presence of smooth muscle cell actin (31). Cells were grown in modified Eagle's Medium (GIBCO BRL, Gaithersburg, MD) supplemented with $10 \%$ fetal calf serum (Gemini, Calabasas, CA) and antibiotics as described $(30,32)$. Cultures used were from passages 4-8. When smooth muscle cells achieved confluence in 24-well plates, the medium was aspirated and fresh growth medium was added just before exposing cultures to normoxia (ambient air) or normobaric hypoxia in a specially designed hypoxia chamber (Coy Laboratory Products, Ann Arbor, MI). Use of this chamber has been described in detail previously $(21,32)$, and the $\mathrm{PO}_{2}$ of the medium was $\sim 15-20$ Torr, assessed using a dissolved gas analyzer (model ABL-2 Radiometer, Copenhagen, Denmark). Cells were exposed to hypoxia or normoxia for the indicated times, aliquots of conditioned medium were harvested, cells were washed twice with phosphate-buffered saline, and a third time in the same buffer containing 3-isobutyl-1-methylxanthine (Sigma Chemical Co.). Cultures were then lysed by the addition of ice-cold trichloroacetic acid $(6 \%)$. The trichloroacetic acid-soluble supernatant was removed from the well, extracted five times with water-saturated ether, dried, and the pellet was resuspended in sodium acetate buffer ( $\mathrm{pH} 6.2)$. A radioimmunoassay was then performed for cAMP according to the manufacturer's instructions (New England Nuclear, Boston, MA), as we have reported previously (25). Protein content of smooth muscle cell cultures was determined by the method of Lowry (33) after solubilizing protein precipitated in trichloroacetic acid in the 24-well plates with sodium dodecyl sulfate $(2 \%)$. The protein content of hypoxic smooth muscle cell cultures was within $10 \%$ of the value for their normoxic counter- 
parts. Cell viability was determined throughout the exposure to hypoxia by trypan blue exclusion, morphologic criteria, and release of lactate dehydrogenase into the cultured medium (the latter using a kit obtained from Sigma Chemical Co.).

Adenylate cyclase activity was determined using SMCs grown to confluence in $10 \mathrm{~cm}^{2}$ culture dishes exposed to either normoxia (standard cell incubator under ambient conditions) or hypoxia for $12 \mathrm{~h}$ at $37^{\circ} \mathrm{C}$. Cells were washed three times with ice-cold $0.9 \%$ saline, and scraped into $30 \mathrm{ml}$ of buffer containing $0.25 \mathrm{M}$ sucrose, $50 \mathrm{mM}$ Tris (hydroxymethyl)aminomethane (Tris): $\mathrm{HCl}(50 \mathrm{mM}, \mathrm{pH}, 7.4), 5$ $\mathrm{mM} \mathrm{MgCl} 2,5 \mathrm{mM}$ EDTA, $2 \mathrm{mM}$ DTT and $100 \mu \mathrm{M}$ PMSF. Cell suspensions were homogenized using a Polytron homogenizer at half maximal speed for $30 \mathrm{~s}$, and used immediately in an adenylate cyclase assay as previously described (34). Briefly, 25- $\mu$ l aliquots of the homogenate were added to $40 \mu \mathrm{l}$ of a reaction mixture containing $50 \mathrm{mM}$ Tris- $\mathrm{HCl}$ ( $\mathrm{pH} 7.4), 2.5 \mathrm{mM} \mathrm{Mg}^{2+}, 0.143 \mathrm{mM}$ ATP, an ATP regenerating system (creatine phosphate/creatine phosphokinase), ATP ([ $\left.\alpha-{ }^{32} \mathrm{P}\right] \mathrm{ATP}$, 1-2 $\times 10^{6} \mathrm{cpm} /$ assay tube [New England Nuclear]), and $6 \mathrm{mM}$ theophylline (Sigma Chemical Co., incubated in a shaking water bath at $37^{\circ} \mathrm{C}$ for $15 \mathrm{~min}$. The adenylate cyclase reaction was terminated by the addition of an ATP-cAMP "stopping solution" as described (35). Isolation of $\left[{ }^{32} \mathrm{P}\right]$ cAMP was accomplished by sequential Dowex and alumina chromatography with $\left[{ }^{3} \mathrm{H}\right]$ cAMP as a recovery marker (36). Protein was determined as described above, and activity was expressed as picomoles of CAMP/15 min per milligram of protein.

Phosphodiesterase activity was measured by preparing cell homogenates as described for adenylate cyclase, centrifuging at $40,000 \mathrm{~g}$ for 20 $\mathrm{min}$, and then analyzing the supernatant for phosphodiesterase activity using the two-step method as previously described (37). Subtypes of phosphodiesterase were calculated as the difference between total and inhibited phosphodiesterase activities using specific inhibitors (37). Initial studies (not shown) confirmed that the reaction conditions employed were linear within the time period of the assay.

Statistics. Graft survival data and transplant scores were analyzed using the Kruskal-Wallis nonparametric analysis of variance (38). Comparisons of individual treatments were performed using the procedure described by Dunn (39). In addition, the effect of db-cAMP on the time-course of graft failure was quantified by multivariate logistic regression. Graft myeloperoxidase data, microsphere data, and cAMP/ adenylate cyclase/phosphodiesterase data from experiments with cultured vascular smooth muscle cells were analyzed by $t$ tests when two treatments were performed or by analysis of variance when three or more treatments were performed. In the latter case, post hoc comparison of treatment groups were tested using Tukey's procedure. Values are expressed as mean \pm SEM, with a $P<0.05$ considered statistically significant.

\section{Results}

Enhanced cardiac preservation in the presence of CAMP analogues/agonists in a heterotopic rat transplant model. Hearts preserved in LR alone showed a decline in graft survival and scores that were dependent on the preservation time, with two thirds of grafts nonfunctional when preserved $>4 \mathrm{~h}$, and $93 \%$ failing when preservation duration was $12 \mathrm{~h}$ (Fig. $1 \mathrm{~A}$ ). Addition of the cyclic AMP analogue db-cAMP to LR solution prolonged graft survival and improved graft scores, resulting in $90 \%$ survival at $12 \mathrm{~h}$ compared with $7 \%$ survival with LR alone. The beneficial effect of db-cAMP was dose dependent, with optimal preservation occurring at 1-2 mM (Fig. $1 B$ ) (heart transplant survival closely paralleled transplant score in these and subsequent experiments, so that only the graft survival is shown in subsequent figures).

To establish that the cAMP was responsible for improving graft survival, and knowing that when db-cAMP enters the cell it is converted to $N^{6}$-monobutyryladenosine $3^{\prime}, 5^{\prime}$ monophosphate
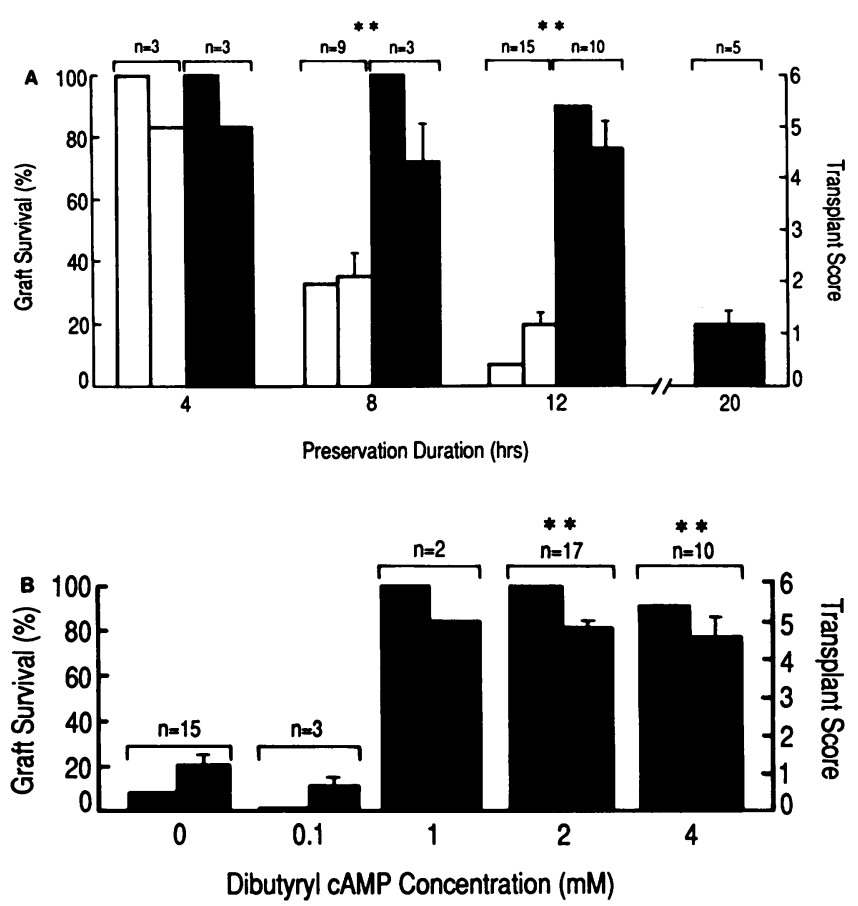

Figure 1. Effect of db-cAMP on cardiac preservation in LR. $(A)$ Dependence on preservation time. Hearts were explanted, flushed with cold LR alone (open bars) or LR + db-cAMP ( $4 \mathrm{mM}$; closed bars), and then stored in the same solution at $4^{\circ} \mathrm{C}$ for the indicated times. Data are reported (as described in the text) as percent survival (left bar) or transplant score (right bar), both assessed at 10 min after release of the aortic cross-clamp. Db-cAMP prolongs the duration of preservation that is associated with subsequent successful transplantation. $(B)$ Dependence on db-cAMP concentration in the preservation solution. Hearts were explanted and stored for $12 \mathrm{~h}$ as above in LR supplemented with the indicated concentration of db-cAMP ( $\mathrm{mM}$ ). The percent survival (left bar) and cardiac score (right bar) are shown. $n$ is indicated for each point. Note that the 12-h control preservation group (LR) displayed in these panels is also shown in Fig. 2 for illustrative purposes; the nonparametric analysis of variance (see Methods) takes this into account in calculating significance of the data. ${ }^{* *} P<0.01, \mathrm{LR}+\mathrm{db}$-cAMP vs LR alone.

and butyrate (40), the effect of sodium butyrate alone was assessed (Fig. $2 \mathrm{~A}$ ). Hearts preserved with butyrate in place of $\mathrm{db}$-cAMP consistently failed after a $12-\mathrm{h}$ preservation period. 8-Br-cAMP, another cAMP analogue, prolonged graft survival, whereas 8-bromoadenosine was ineffective. When 8-Br-cAMP was tested over a broad range of concentrations during a 12-h preservation period, it was effective at concentrations $\sim 10$ times lower than db-cAMP (Fig. $2 B$ ): $100 \%$ of grafts survived when preserved in LR solution containing 8-Br-cAMP at 0.1 $\mathrm{mM}$. Further evidence in support of cyclic AMP's role in successfully prolonging the preservation period come from the results of experiments in which the phosphodiesterase inhibitors rolipram $(10 \mu \mathrm{M})$ or indolidan $(10 \mu \mathrm{M})(41)$ uniformly enhanced preservation of hearts stored for $12 \mathrm{~h}$ in LR solution (Fig. $2 C$ ). These data suggest that elevation of endogenous cAMP levels has a similar beneficial effect to exogenously administered cyclic AMP analogue.

An important means through which cAMP exerts its effects intracellularly is via stimulation of the cAMP-dependent protein kinase (PKA). To explore the potential relevance of this 

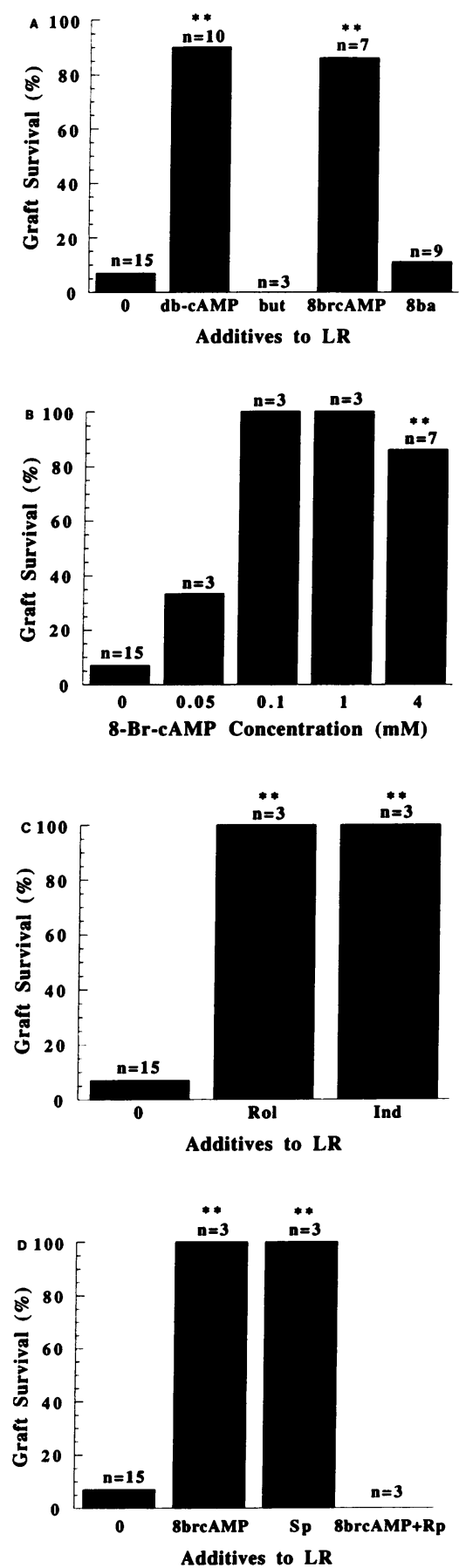
AMP-dependent protein kinase agonist $S_{p}$-cAMPS $(0.5 \mathrm{mM})$ was
added to LR. Bars represent the percentage of surviving grafts and demonstrate the importance of the cAMP-dependent protein kinase with respect to preservation. ${ }^{* *} P<0.01$ compared with LR alone; preservation scores paralleled survival data in all instances and are not shown.

mechanism to enhanced cardiac preservation, two stereoisomers of adenosine 3',5'monophosphorothioate were used (42). $\mathrm{S}_{\mathrm{p}}$-cAMPS is a PKA agonist that is not hydrolyzable by cellular enzymes, whereas $R_{p}$-cAMPS is a nonhydrolyzable competitive PKA antagonist, which binds to the PKA without causing activation. $S_{p}$-cAMPS enhanced preservation, whereas $R_{p}$ -
cAMPS abolished the salutory effect of 8-Br-cAMP on preservation, consistent with the important role of the PKA pathway in preservation enhancement (Fig. $2 \mathrm{D}$ ). Although the cyclic GMP-dependent protein kinase may have a role in cardiac preservation, its direct activation by cyclic AMP analogues is unlikely, since $N^{6}$-monobutyryladenosine $3^{\prime}, 5^{\prime}$ monophosphate and 8-Br-cAMP are, respectively, 313-fold and 53-fold less potent than cGMP in activating the cGMP-dependent protein kinase (43), and $S_{p}$-cAMPS is an antagonist of the cGMP-dependent protein kinase (44).

Because the simple balanced salt solution (LR) that was used for these initial experiments is not used clinically for cardiac preservation, it was important to determine whether a cAMP analogue enhanced preservation using the clinical standard, UW solution $(4,45)$. Addition of db-cAMP to UW solution increased the time for effective preservation (Fig. $3 \mathrm{~A}$ ), with $75 \%$ of hearts surviving after $24 \mathrm{~h}$ of storage in $\mathrm{UW}+\mathrm{db}-$ cAMP vs only $35 \%$ in UW alone $(P<0.05)$. As with $L R$, the beneficial effects of db-cAMP on cardiac preservation in UW occurred in a dose-dependent fashion (Fig. $3 \mathrm{~B}$ ).

Effect of CAMP analogs added to cardiac preservation solution on graft perfusion and leukocyte infiltration. Because the hypothesis that CAMP analogues enhanced cardiac preservation was borne out by the experiments described above, and knowing that elevated intracellular cAMP levels in vascular smooth muscle cells is associated with vasorelaxation (27), we evaluated the effects of cAMP analogues on graft blood flow after transplantation. Relative blood flow was assessed by the injection of colored latex microspheres into the aortic root of the transplanted heart, and quantitating deposition of microspheres $(10 \mu \mathrm{m})$ in the vasculature (46). Histologic studies demonstrated increased numbers of beads trapped in the microvasculature of hearts that were successfully transplanted, indic-

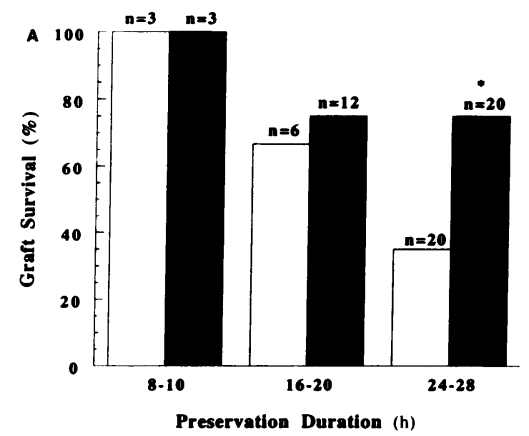

Figure 3. Db-cAMP enhances cardiac preservation in UW solution. $(A)$ Dependence on preservation time. Hearts were explanted and preserved (as described above) with UW (open bars) or UW + db-cAMP ( 4 mM, closed bars) for the indicated times. The percentage of grafts surviving was evaluated 10 min after release of the aortic cross-clamp as described in the text. $(B)$ Dependence on dbcAMP concentration. Hearts were explanted and preserved for $12 \mathrm{~h}$ with UW supplemented with the indicated concentrations of dbcAMP. Graft survival is plotted vs the concentration of db-cAMP added to the preservation solution. ${ }^{*} P<0.05, \mathrm{UW}+\mathrm{db}$-cAMP vs UW alone. 
ative of increased perfusion, vs fewer beads in grafts that failed. When hearts preserved in LR alone were compared with those preserved with LR + db-cAMP $(2 \mathrm{mM})$ for $12 \mathrm{~h}$, a significant increase in blood flow was observed in those hearts preserved with supplemental db-cAMP (Fig. 4).

Infiltration of the heart by PMNs is an important cause of myocardial damage in the setting of ischemia/reperfusion (47-53). Consistent with this view, histologic examination of the vasculature from a heart preserved in LR, which subsequently failed, showed a ring of PMNs adherent to the vessel surface, which was not seen in a successfully transplanted cardiac graft preserved in LR + db-cAMP (Fig. $5 A$, panels $I-I I$ ). This was quantified by measuring myeloperoxidase activity in grafts as an index of the presence of PMNs. Hearts preserved for $12 \mathrm{~h}$ in LR + db-cAMP ( $2 \mathrm{mM}$ ) showed a 3.2-fold decrease in myeloperoxidase activity compared with controls preserved for $12 \mathrm{~h}$ in LR alone $(P<0.05)$.

Effect of hypoxia on vascular smooth muscle cell cAMP. The effect of db-cAMP to increase perfusion in the cardiac graft suggested the possibility that it was acting at the level of the vascular smooth muscle cell to induce vasorelaxation. One mechanism underlying this observation would be if harvest and storage of the heart resulted in hypoxia and an associated fall in smooth muscle cell cAMP, as occurs in hypoxic ECs (25), thereby preventing normal vasorelaxation in the graft. Supporting this hypothesis is the fact that the measured $\mathrm{PO}_{2}$ of the storage solution remaining in the vasculature of a preserved heart for $18 \mathrm{~h}$ was extremely hypoxic (19.9 Torr). Exposure of cultured smooth muscle cells to hypoxia resulted in a fall in intracellular cAMP levels (Fig. 6 A, closed bars). This occurred in a time-dependent manner when cultures were exposed to an atmosphere with $\mathrm{Po}_{2} \sim 15-20$ Torr, with cAMP levels falling from $8.4 \pm 0.9 \mathrm{pg} / \mathrm{ml}$ per $\mathrm{mg}$ cell protein during normoxia to $3.0 \pm 0.6 \mathrm{pg} / \mathrm{ml}$ per $\mathrm{mg}$ cell protein after $12 \mathrm{~h}$ of hypoxic conditions. This decline could not be attributed to extrusion of cAMP into the growth medium (Fig. $6 \mathrm{~A}$, open bars), or to cell death, as smooth muscle cells remained firmly attached to the growth surface (there was no increase in floating cells), there

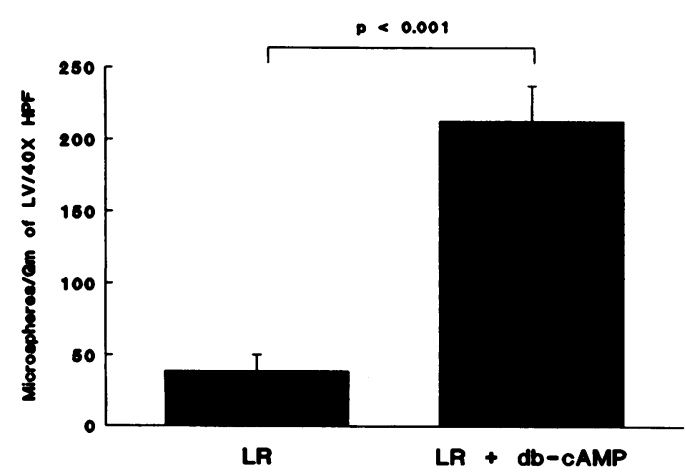

Figure 4. Blood flow as measured by accumulation of colored latex microspheres in graft vasculature. Hearts were preserved for $12 \mathrm{~h}$ and transplanted; $10 \mathrm{~min}$ after the release of the aortic cross-clamp, a suspension of latex microspheres $\left(0.5 \mathrm{ml}, 10^{6} / \mathrm{ml}\right)$ was injected into the donor aortic root, and the heart was excised after $1 \mathrm{~min}$. Microspheres were recovered from ventricular tissue after alkaline hydrolysis, counted, and expressed as microspheres/gram per $\times 40$ highpower field (mean of 10 observations \pm SEM is shown. Preservation solutions included LR alone $(n=5)$ or LR + db-cAMP ( $2 \mathrm{mM}, n$ $=10$ ). Addition of db-cAMP to the preservation solution is associated with increased graft blood flow. was no increase in trypan blue uptake, and lactate dehydrogenase levels in the medium were not elevated (data not shown).

To determine whether decreased production or increased degradation of cAMP was responsible for the hypoxia-induced decline in intracellular cAMP in vascular smooth muscle cells, adenylate cyclase and phosphodiesterase (PDE) activities were measured. There was no measurable difference in baseline (or stimulated) adenylate cyclase activity between normoxic and hypoxic smooth muscle cells (Fig. $6 \mathrm{~B}$ ). In contrast, hypoxia augmented the metabolism of CAMP. Although total PDE activity was not significantly increased in hypoxia $(82 \pm 4.7 \mathrm{nmol}$ adenosine / $\mathrm{min}$ per $\mathrm{mg}$ protein vs $77.1 \pm 4.4$ for hypoxia vs normoxia, respectively), there were significant increases in both types III and IV PDE activity (59.2 and $30.0 \%$ increase, respectively, $P<0.001$ and $P<0.05$ for types III and IV PDE, respectively) (Fig. $6 C$ ). These results suggest that the decline in vascular smooth muscle cell cAMP is not caused by a decreased production, but rather, to an increased activity of the enzyme responsible for its degradation.

\section{Discussion}

These studies show that addition of agents which elevate intracellular cAMP to cardiac preservation solutions increases the maximal duration of cold ischemia associated with restoration of cardiac graft function after transplantation in a rat heterotopic cardiac transplant model. The cAMP analogues used in our studies appear to exert their effect(s) via stimulation of cyclic AMP-dependent protein kinase, presumably followed by recruitment of effector mechanisms, although the possible involvement of the cyclic GMP-dependent protein kinase cannot be ruled out. Furthermore, cAMP analogues enhanced preservation when added to UW solution, the clinically accepted standard for cardiac preservation $(4,45)$. Second messenger cyclic nucleotides such as cAMP have diverse biologic effects, and it is likely that many cell types within the heart are effected by cAMP analogues in the preservation solution. In preliminary studies, we have determined that the resting membrane potential of individual myocytes is more negative when a heart has been preserved with a preservation solution containing dbcAMP and nitroglycerin, compared to a preservation solution without these agents (54). Although this effect on nonvascular cells within the heart may contribute to the beneficial effects of cAMP analogues on graft survival, the analysis of hearts in the current study focuses on markers of graft vascular function. These studies indicate that agents which stimulate the cAMP second messenger pathway maintain blood flow, diminish leukostasis, and preserve tissue turgor and color after reperfusion. These observations underscore the role of the cAMP pathway and the importance of maintaining vascular homeostasis within the graft for the success of organ transplantation.

The period of reperfusion is an especially vulnerable period for the graft with respect to maintenance of vascular function, because restoration of blood flow initiates the host response through the interaction of leukocytes, the coagulation and complement systems, with the hypoxic/reoxygenated endothelium. In these experiments, grafts were evaluated at $10 \mathrm{~min}$ of reperfusion for several reasons. First, pilot studies demonstrated that transplanted hearts started with a pale color immediately out of preservation solution but turned pink after release of the aortic cross-clamp, as blood could be visibly ob- 

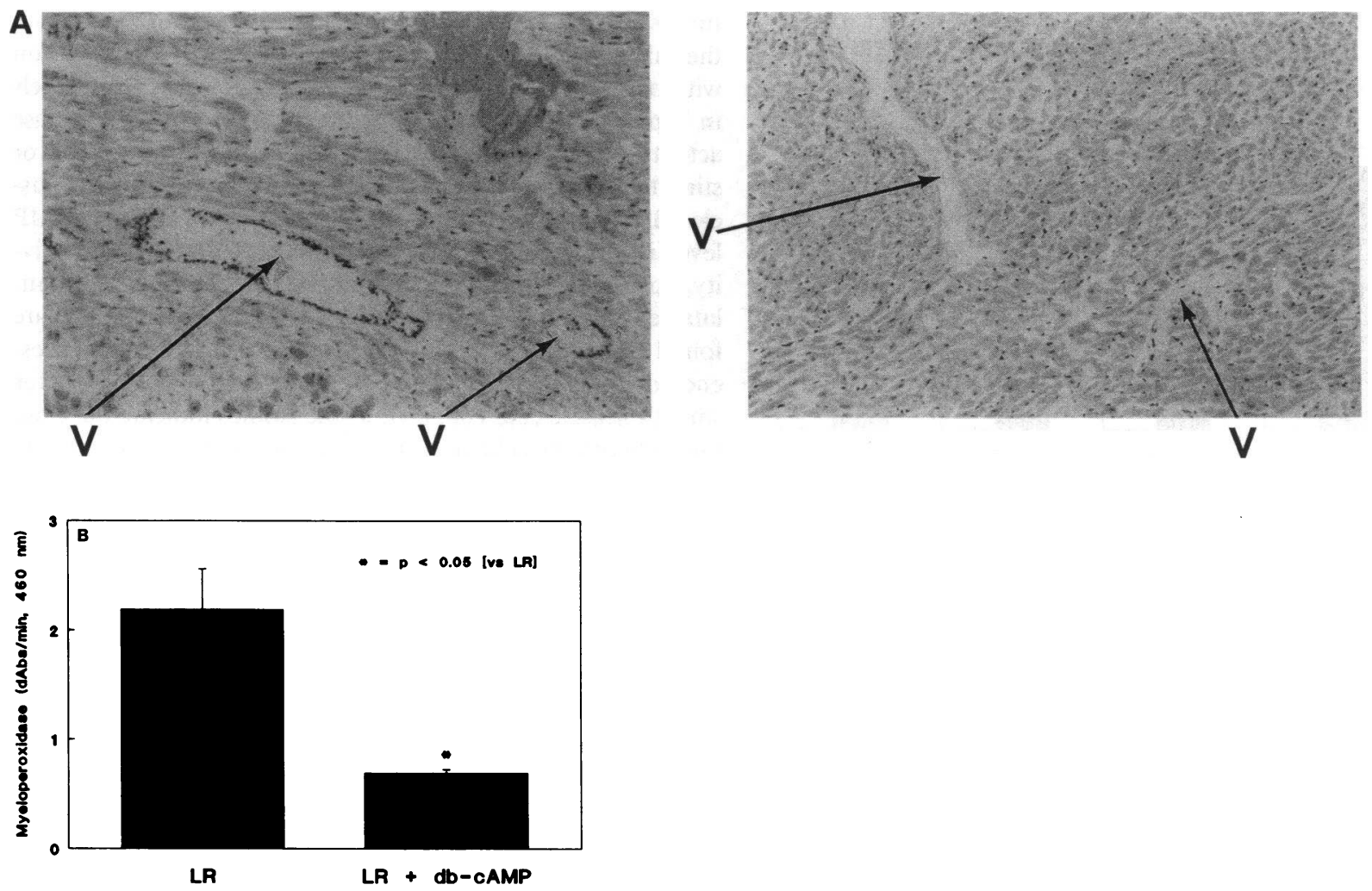

Figure 5. (A) Leukostasis in the transplanted heart $10 \mathrm{~min}$ after restoration of blood flow. Hearts preserved successfully for $12 \mathrm{~h}$ with db-cAMP $(2$ $\mathrm{mM}$ ) in LR (right panel) show no PMNs in a blood vessel $(V)$, whereas many adherent PMNs are seen forming a ring about the luminal surface of vessels from a failed graft preserved for $12 \mathrm{~h}$ with LR alone (left panel). Sections were stained with hematoxylin and eosin. $\times 200$. (B) Leukocytic infiltration after preservation/transplantation. Myeloperoxidase (MPO) activity was measured in tissue homogenates from cardiac grafts harvested $10 \mathrm{~min}$ after the reestablishment of blood flow. Grafts preserved for $12 \mathrm{~h}$ in LR + db-cAMP $(2 \mathrm{mM}, n=3)$ had lower levels of MPO activity compared with grafts stored in LR alone $(n=6)$, which had 3.2-times more MPO activity per gram of protein.

served to distend the epicardial coronary arteries. Hearts that failed always followed this initial sequence, but 2-3 min after the start of reperfusion, would darken and become palpably turgid, and fail to contract. Failed grafts never regained function regardless of the observation duration, whereas surviving grafts continued to function for several hours of observation. A blinded analysis of graft function at $10 \mathrm{~min}$ was never altered by a subsequent evaluation of the same graft as late as $8 \mathrm{~h}$ later. These results are consonant with experiments using coronary artery rings from feline hearts subjected to ischemia/reperfusion in vivo, which demonstrate impaired vasorelaxation within minutes of reperfusion (19). In addition, toxic oxidants formed within minutes of reoxygenation/reperfusion in endothelial cells $(55,56)$ and hearts $(57,58)$ may contribute to the pathogenesis of vascular dysfunction in the reperfused graft. Because these oxidants form early after reperfusion and can alter vascular permeability and lower cAMP levels (59), grafts were evaluated $10 \mathrm{~min}$ after the start of reperfusion.

Although morphologic evidence of parenchymal and vascular damage is subtle during the period of organ storage/hypoxia (data not shown), in contrast to the pathologic changes observed during reperfusion, the period of hypoxia is emerging as an important priming factor for subsequent vascular dysfunction. Hypoxic ECs have enhanced thrombogenicity (21, 60-62), leukocyte adhesivity $(63,64)$, and monolayer permeability (25), and are primed for the production of oxygen free radicals $(55,58)$. In this context of vascular homeostasis, the cAMP second messenger system has multiple roles. In ECs, decreased cAMP levels result in perturbation of cell shape and decreased barrier function of the monolayer $(22,24,25)$, whereas increased levels of cAMP block induction of EC procoagulant activity (23), cause induction of the anticoagulant cofactor thrombomodulin (23), and block induction of transcription of certain leukocyte adherence molecules in response to the cytokine tumor necrosis factor (65). In vascular smooth muscle, increased cAMP levels are associated with vasorelaxation (27). In PMNs, elevated levels of cAMP are associated with decreased EC-leukocyte interactions (66), as well as diminished production of superoxide (67). Thus, addition of agents such as CAMP analogues to organ preservation solutions to preserve vascular homeostasis may prove to be important for prolonging preservation and minimizing posttransplantation organ dysfunction.

To explore the role of cAMP, hypoxia was used to simulate a significant component of ischemia, and cardiac transplantation was used as a model of global ischemia and reperfusion. The observations in this study emphasize the potential importance of suppression of endogenous cAMP levels in the pathogenesis of functional abnormalities associated with ischemia/ reperfusion. Hypoxia-induced suppression of intracellular cAMP in cultured ECs and smooth muscle cells underscores the relevance of this intracellular messenger when the vascula- 

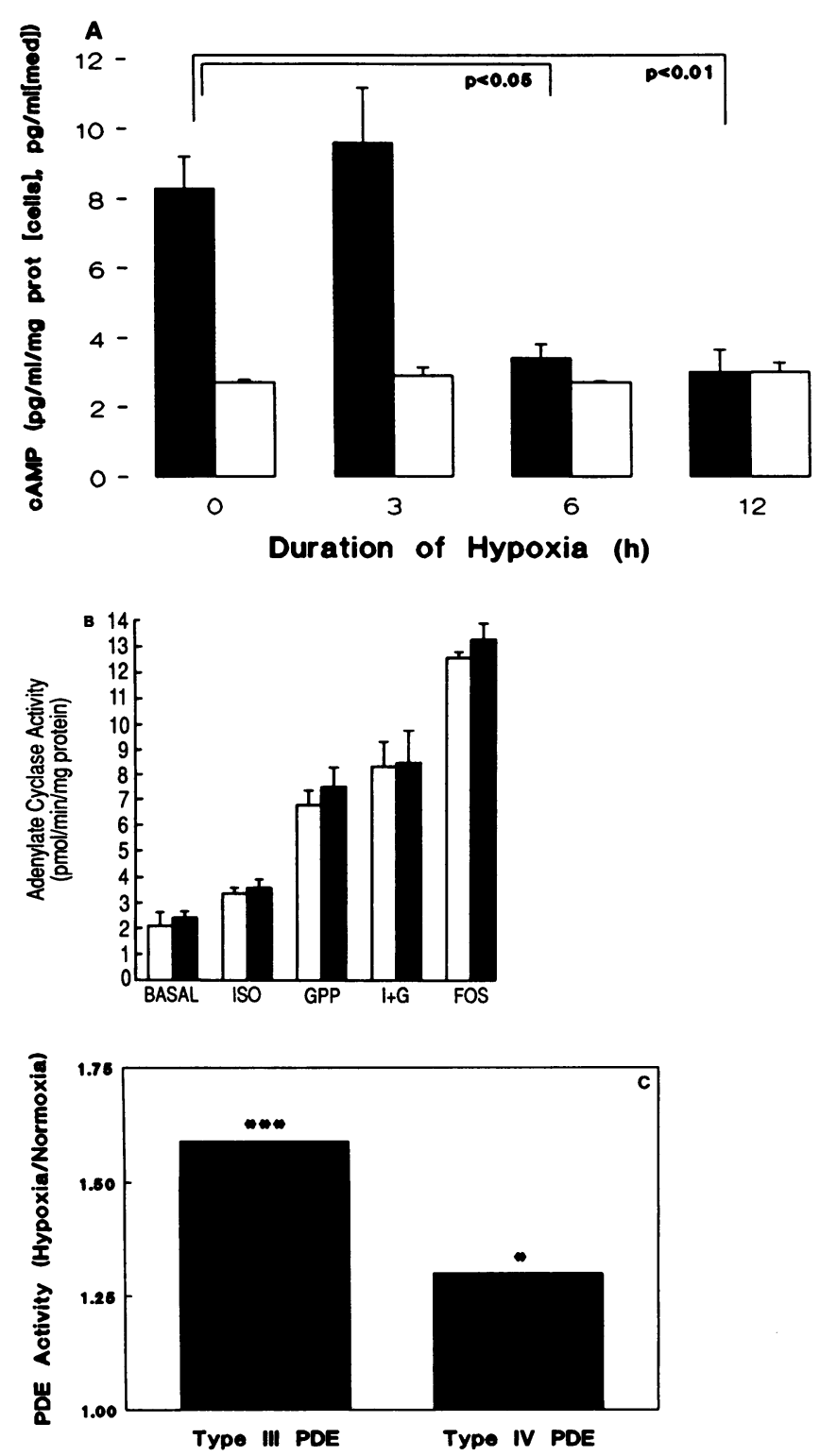

Figure 6. Effects of hypoxia on cultured vascular smooth muscle cell cAMP levels, generation, and degradation. $(A)$ Time-course of cAMP decline in vascular smooth muscle cells exposed to a hypoxic environment $\left(\mathrm{PO}_{2} 15-20\right.$ Torr $)$. Confluent smooth muscle cells were exposed to a hypoxic environment for the indicated durations, and cell lysates (closed bars) and conditioned medium (open bars) were analyzed for cAMP content by radioimmunoassay. cAMP levels decline in parallel with the duration of exposure to the hypoxic environment, without evidence of enhanced extrusion into the culture medium. (B) Adenylate cyclase activity of homogenates vascular smooth muscle cells exposed to $12 \mathrm{~h}$ of hypoxia (closed bars) or normoxia (open bars) at baseline $(B A S A L)$, or with added isoproterenol $(10 \mu \mathrm{M}$; $I S O)$; $\mathrm{Gpp}(\mathrm{NH}) \mathrm{p}(10 \mu \mathrm{M} ; G P P)$; the combination of isoproterenol and $\mathrm{Gpp}(\mathrm{NH}) \mathrm{p}(I+G)$; or forskolin $(10 \mu \mathrm{M} ; F O S)$. Basal and stimulated adenyl cyclase activities are similar for both normoxic and hypoxic vascular SMCs. $(C)$ Phosphodiesterase activity of homogenates of normoxic and hypoxic vascular smooth muscle cells. Total phosphodiesterase activity (normalized to protein concentration) in the soluble (cytosolic fraction) of vascular SMCs exposed to $12 \mathrm{~h}$ of normoxia or hypoxia was determined in the absence of inhibitors of PDE, as well as in the presence of indolidan ( $1 \mu \mathrm{M}$; type III PDE inhibitor) or rolipram ( $10 \mu \mathrm{M}$; type IV PDE inhibitor). Types III and IV PDE activities were calculated as the difference between total and ture is exposed to hypoxia, as occurs during prolonged hypothermic organ storage in the absence of continuous perfusion with oxygenated buffer. Although the decline in cAMP levels in hypoxic ECs results from suppression of adenylate cyclase activity, hypoxia does not substantially alter either basal or stimulated adenylate cyclase activity in vascular smooth muscle cells. Our data indicate that this decrease in cellular cAMP levels is associated with a specific increase in soluble PDE activity, notably types III (cGMP inhibited) and IV (cAMP stimulatable) PDE activity (41). Both types III and IV PDE are found in cultured smooth muscle cells (68); indeed, the presence of soluble type III appears to be relatively specific for smooth muscle cells $(68,69)$. These studies indicate the close link between the cellular response to an environment with low oxygen concentrations and cAMP metabolism.

The beneficial role of PDE inhibition during transplantation is underscored by its importance in other models of ischemia and reperfusion. A recent study (67) in a canine model of ischemia/reperfusion has shown that although rolipram administration does not limit myocardial infarct size, it significantly reduces neutrophil production of superoxide in vitro and limits the "no reflow" phenomenon, wherein coronary blood flow progressively declines after infarction/reperfusion, possibly because of the accumulation of neutrophil aggregates (70-72). Consistent with these observations, a rabbit model of global myocardial ischemia and reperfusion demonstrates that indices of left ventricular performance are improved and histologic evidence of edema and myocardial capillary injury are lessened by either depleting blood of leukocytes or administering a PDE inhibitor before the onset of reperfusion (73), and a feline model of stroke demonstrates that inhibition of PDE in vascular smooth muscle is associated with enhanced restoration of blood flow after release of occlusion of the middle cerebral artery (74).

Although the interplay of cAMP and cGMP in smooth muscle cells has yet to be determined, it was recently proposed (75) that a decrease in smooth muscle cGMP content may mediate the observed hypoxic contraction of isolated rat pulmonary arteries. In addition, ischemia/reperfusion induced declines in nitric oxide levels secondary to the quenching effect of oxygen free radicals $(58,76,77)$ would lower the intracellular cGMP content of vascular SMCs, which might, in turn, cause less inhibition of the type III PDE. Thus, increased levels as well as disinhibition of smooth muscle (type III) PDE would amplify the destruction of intracellular cAMP, accounting for the observed decrease in cAMP levels.

Because of these interactions between the cAMP and the cGMP second messenger systems, as well as recent studies which have demonstrated diminished production of nitric oxide by hypoxic/reoxygenated ECs (Pinsky, D., and D. Stern, unpublished observation ) and reduced agonist induced nitric oxide generation by UW-preserved hearts (78), it is likely that supplementation of the intra-intercellular second messenger pathway involving nitric oxide/cGMP will also enhance vascular homeostasis in the setting of organ transplantation. Our

inhibited PDE activities, measured in the presence of indolidan or rolipram, respectively. The graph represents the means of three determinations each for normoxic and hypoxic samples, and demonstrates an increase in both types III and IV PDE activity in the hypoxic SMCs. 
recent pilot studies have provided support for this hypothesis in a rat heterotopic transplant model (79). Because the cAMP and nitric oxide/cGMP pathways share many similar features with respect to vascular homeostasis (77), they may provide synergistic benefit during the preservation and reimplantation periods. Consistent with this hypothesis, supplementation of both the cyclic AMP and nitric oxide/cGMP messenger pathways results in unprecedented enhancement of cardiac preservation ( $24 \mathrm{~h}$ with simple hypothermic storage) in a primate orthotopic model involving cardiopulmonary bypass (80), indicating the relevance of this approach with respect to human heart transplantation, as well as other situations involving ischemia and reperfusion. Based on these considerations, we propose that intervention at the level of intra-intercellular second messenger pathways during cardiac preservation and reimplantation provides a new approach to the problem of organ preservation. The clinical benefits that should accrue from successfully prolonging the preservation period include more time for better immunologic cross-matching (with attendant reduction in immunologic complications), as well as expanding the donor and recipient pools by reducing geographic constraints, thereby making transplantation more widely available as a therapeutic option.

\section{Acknowledgments}

We thank A. Morales for expert assistance with these studies, Drs. H. Spotnitz and $R$. Kessin for providing invaluable suggestions during the course of this work, and Dr. G. Godman provided detailed critiques of the manuscript.

This work was support by grants from the Public Health Service (HL-34625, HL-42833, HL-42507, and HL-21006), the Council for Tobacco Research, the Stony-Wold Foundation, the New York Heart Association, and the Surgical Research Fund. D. Stern completed this work during the tenure of a Genentech-EI Award from the American Heart Association. D. Pinsky was supported by the Atherosclerosis Training grant (HL-07343).

\section{References}

1. Roberts, A. 1985. Myocardial Protection in Cardiac Surgery. Marcel Dekker, Inc., New York.

2. Wahlberg, J., J. Southard, and F. Belzer. 1986. Development of a cold storage solution for pancreas preservation. Cryobiology. 23:477-482.

3. Makowka, J., T. Zerbe, and F. Chapman. 1989. Prolonged rat cardiac preservation with UW lactobionate solution. Transplant. Proc. 21:1350-1352.

4. Swanson, D., I. Pasaoglu, H. Berkoff, J. Southard, and J. Hegge. 1988. Improved heart preservation with UW preservation solution. J. Heart Transplant. 7:456-467.

5. Malhotra, D., A. Zhou, Y. Kong, J. Shapiro, and L. Chan. 1991. Improvement in experimental cardiac preservation based on metabolic considerations. Transplantation (Baltimore). 52:1004-1008.

6. Wickens, D., M. Li, G. Atkins, B. Fuller, K. Hobbs, and T. Dormandy. 1987. Free radicals in hypothermic rat heart preservation-prevention of damage by mannitol and desferrioxamine. Free Rad. Res. Commun. 4:189-195.

7. Belzer, F., and J. Southard. 1988. Principles of solid-organ preservation by cold storage. Transplantation (Baltimore) . 45:673-676.

8. Warnick, C., and H. Lazarus. 1977. Adenine nucleotides during organ storage. Transplant. Proc. IX:1575-1577.

9. Stewart, J., W. Frist, and W. Merrill. 1990. Oxygen scavengers in myocardial preservation during transplantation. Methods Enzymol. 186:742-748.

10. Das, D., J. Russell, and R. Jones. 1991. Reduction of cold injury by superoxide dismutase and catalase. Free Rad. Res. Commun. 12-13 (Pt. 2):653662 .

11. Zimmer, S., K. Ugurbil, S. Michurski, P. Mohanakrishnan, V. Ulstad, J. Foker, and A. From. 1989. Alterations in oxidative function and respiratory regulation in the post-ischemic myocardium. J. Biol. Chem. 12402-12411.

12. Oksendal, A., and P. Jynge. 1986. Protection by verapamil in the calcium paradox: dependence on micromolar calcium. Cardiovasc. Res. 20:845-852.
13. Boggs, B., D. Torchiana, G. Geffin, J. Titus, B. Redonnett, D. O'Keefe, J. Newell, and W. Daggett. 1987. Optimal myocardial preservation with an acalcemic crystalloid cardioplegic solution. J. Thoracic. Cardiovasc. Surg. 93:838-846.

14. Iyengar, J., A. George, J. Russell, and D. Das. 1990. The effects of an iron chelator on cellular injury induced by vascular stasis caused by hypothermia. $J$. Vasc. Surg. 12:545-551.

15. Liu, X., Engelman, R., J. Iyengar, G. Cordis, and D. Das. 1991. Amiloride enhances postischemic ventricular recovery during cardioplegic arrest. Ann. NY Acad. Sci. 639:471-474.

16. Simionescu, N., and M. Simionescu. 1988. Endothelial Cell Biology. Plenum Publishing Corp., New York.

17. Simionescu, N., and M. Simionescu. 1991. Endothelial Cell Dysfunction. Plenum Publishing Corp., New York.

18. Shreeniwas, R., S. Ogawa, F. Cozzolino, G. Torcia, N. Braunstein, C. Butura, J. Brett, H. Lieberman, M. Furie, and D. Stern. 1991. Macrovascular and microvascular endothelium during long-term hypoxia: alterations in cell growth monolayer permeability, and cell surface coagulant properties. J. Cell. Physiol. 146:8-17.

19. Lefer, A., P. Tsao, D. Lefer, and X. Ma. 1991. Role of endothelial dysfunction in the pathogenesis of reperfusion injury after myocardial ischemia. FASEB (Fed. Am. Soc. Exp. Biol.) J. 5:2029-2034.

20. Minnear, F., A. Johnson, and A. Malik. 1986. $\beta$-adrenergic modulation of pulmonary transvascular fluid and protein exchange. J. Appl. Physiol. 60:266274.

21. Ogawa, S., H. Gerlach, C. Esposito, A. Pasagian-Macaulay, J. Brett, and D. Stern. 1990. Hypoxia modulates the barrier and coagulant function of cultured bovine endothelium. Increased monolayer permeability and induction of procoagulant properties. J. Clin. Invest. 85:1090-1098.

22. Hoek, J. 1992. Intracellular signal transduction and the control of endothelial permeability. Lab. Invest. 67:1-4.

23. Archipoff, G., A. Beretz, C. Froger-Leon, C. de la Salle, C. Klein-Soyer, and $\mathrm{H}$. Cazenave. 1991. Opposite regulation by cAMP of thrombomodulin and tissue factor activities on the surface of human saphenous vein endothelial cells in culture. Thromb. Haemostasis. 65:349.

24. Stelzner, T., J. Weil, and R. O'Brien. 1989. Role of cyclic adenosine monophosphate in the induction of endothelial barrier properties. J. Cell. Physiol. 139:157-166.

25. Ogawa, S., S. Koga, K. Kuwabara, B. Morrow, S. Morris, J. Bilezikian, S. Silverstein, and D. Stern. 1992. Hypoxia-induced increased permeability of cultured bovine pulmonary and aortic endothelial monolayers occurs through lowering of cellular cAMP levels and is modulated by glucocorticoids. Am. J. Physiol. 262:C546-554.

26. Seibert, A., W. Thompson, A. Taylor, W. Wilborn, J. Barnard, and J. Haynes. 1992. Reversal of increased microvascular permeability associated with ischemia-reperfusion: role of cAMP. J. Appl. Physiol. 72:389-395.

27. Haynes, J., J. Robinson, L. Saunders, A. Taylor, and S. Strada. 1992. Role of cAMP-dependent protein kinase in CAMP-mediated vasodilation. Am. J. Physiol. 262:H511-516.

28. Ono, K., and Lindsey, E. 1969. Improved technique of heart transplantation in rats. J. Thorac. Cardiovasc. Surg. 57:225-229.

29. Goldblum, S., K. Wu, and M. Jay. 1985. Lung myeloperoxidase as a measure of pulmonary leukostasis in rabbits. J. Appl. Physiol. 59:1978-1985.

30. Colucci, W., T. Brock, M. Gimbrone, and R. Alexander. 1985. Non-linear relationship between $\alpha_{1}$-adrenergic receptor occupancy and norepinephrine-stimulated calcium flux in cultured vascular smooth muscle cells. Mol. Pharmacol. 27:517-524.

31. Gown, A., A. Vogel, D. Gordon, and P. Lu. 1985. A smooth muscle-specific monoclonal antibody recognizes smooth muscle actin isozymes. J. Cell Biol. 100:807-813.

32. Koga, S., S. Ogawa, K. Kuwabara, J. Brett, J. Leavy, J. Ryan, Y. Koga, J. Plocinski, W. Benjamin, D. Burns, and D. Stern. 1992. Synthesis and release of interleukin-1 by reoxygenated human mononuclear phagocytes. J. Clin. Invest. 90:1007-1015.

33. Lowry, O., N. Rosebrough, A. Farr, and R. Randall. 1951. Protein measurement with the Folin reagent. J. Biol. Chem. 193:265-275.

34. Morris, S., H. Tanowitz, S. Factor, J. Bilezikian, and M. Wittner. 1988 Myocardial adenylate cyclase activity in acute murine Chagas' disease. Circ. Res. 62:800-810.

35. Bilezikian, J., A. Dornfeld, and D. Gammon. 1978. Structure activity binding analysis of beta adrenergic amines. Biochem. Pharmacol. 27:1445-1454.

36. Salomon, Y., C. Londos, and M. Rodbell. 1974. A highly sensitive adenylate cyclase assay. Anal. Biochem. 58:541-548.

37. Keravis, T., J. Wells, and J. Hardman. 1980. Cyclic nucleotide phosphodiesterase activities from pig coronary arteries: lack of interconvertibility of major forms. Biochem. Biophys. Acta. 613:116-29.

38. Kruskal, W. H., and W. A. Wallis. 1952. Use of ranks in one-criterion analysis of variance. J. Am. Statist. Assoc. 52:356-360.

39. Dunn, O. J. 1964. Multiple contrasts using rank sums. Technometrics. 6:241-252. 
40. Kaukel, E., K. Mundhenk, and H. Hilz. 1972. $N^{6}$-monobutyryladenosine $3^{\prime}: 5^{\prime}$-monophosphate as the biologically active derivative of dibutyryladenosine 3':5'-monophosphate in HeLa S3 cells. Eur. J. Biochem. 27:197-200.

41. Thompson, W. 1991. Cyclic nucleotide phosphodiesterases: pharmacology, biochemistry, and function. Pharmacol. Ther. 51:13-33.

42. Rothermel, J., L. Botelho, and H. Parker. 1988. A mechanistic and kinetic analysis of the interactions of the diastereoisomers of adenosine $3^{\prime}, 5^{\prime}$-(cyclic) phosphorothioate. Biochem. J. 251:7575-762.

43. Corbin, J., D. Ogreid, J. Miller, R. Suva, B. Jastorff, and S. Doskeland. 1986. Studies of cGMP analog specificity and function of the two intrasubunit binding sites of cGMP-dependent protein kinase. J. Biol. Chem. 261:1208-1214.

44. Hoffman, F., H. Gensheimer, W. Landgraf, R. Hullin, and B. Jastorff 1985. Diastereomers of adenosine 3',5'-monothiophosphonate (cAMP [S])antagonize the activation of the CGMP-dependent protein kinase. Eur. J. Biochem. 150:85-88.

45. Jeevanandam, V., M. Barr, J. Auteri, J. Sanchez, G. Ott, F. Schenkel, C. Marboe, C. Smith, and E. Rose. 1991. University of Wisconsin solution for human donor heart preservation: initial clinical experience. Ann. Thorac. Surg. 52:1213-1216.

46. Hale, S., K. Alker, and R. Kloner. 1988. Evaluation of nonradioactive, colored microspheres for measurement of regional myocardial blood flow in dogs. Circulation. 78:428-434.

47. Mullane, K., and C. Smith. The role of leukocytes in ischemic damage, reperfusion injury and repair of the myocardium. In Pathophysiology of Severe Ischemic Myocardial Injury. H. Piper, editor. Kluwer Academic Publishers Group, Dordrecht, Netherlands. 239-267.

48. Crawford, M., F. Grover, W. Kolb, C. McMahan, R. O'Rourke, L. McManus, and R. Pinckard. 1988. Complement and neutrophil activation in the pathogenesis of ischemic myocardial injury. Circulation. 78:1449-1458.

49. Mullane, K., N. Read, J. Salmon, and S. Moncada. 1984. Role of leukocytes in acute myocardial infarction in anesthetized dogs. J. Pharmacol. Exp. Ther. 228:510-522.

50. Lucchesi, B., and K. Mullane. 1986. Leukocytes and ischemia induced myocardial injury. Annu. Rev. Pharmacol. Toxicol. 26:201-224.

51. Schmid-Schoenbein, G., and R. Engler. 1987. Granulocytes as active participants in acute myocardial ischemia and infarction. Am. J. Cardiovasc. Pathol. 1:15-30.

52. Entman, M., L. Michael, R. Rossen, W. Dreyer, D. Anderson, A. Taylor, and C. Smith. 1991. Inflammation in the course of early myocardial ischemia. FASEB (Fed. Am. Soc. Exp. Biol.) J. 5:2529-2537.

53. Ma, X., P. Tsao, and A. Lefer. 1991. Antibody to CD18 exerts endothelial and cardiac protective effects in myocardial ischemia and reperfusion. J. Clin. Invest. 88:1237-1243.

54. Koga, S., D. Han, R. P. Kline, M. C. Oz, R. Nowygrod, D. M. Stern, and D. J. Pinsky. 1992. Electrical evaluation of myocardial viability following prolonged storage of explanted hearts. Circulation. 86:I-770. (Abstr.)

55. Zweier, J., P. Kuppusamy, and G. Lutty. 1988. Measurement of endothelial cell free radical generation: evidence for a central mechanism of free radical injury in postischemic tissues. Proc. Natl. Acad. Sci. USA. 85:4046-4050.

56. Britigan, B. E., T. L. Roeder, and D. M. Shasby. 1992. Insight into the nature and site of oxygen-centered free radical generation by endothelial cell monolayers using a novel spin trapping technique. Blood. 79:699-707.

57. J. L. Zweier. 1988. Measurement of superoxide-derived free radicals in the reperfused heart. J. Biol. Chem. 263(3):1353-1357.

58. Babbs, C., M. Cregor, J. Turek, and S. Badylak. 1991. Endothelial superoxide production in the isolated rat heart during early reperfusion after ischemia. Am. J. Pathol. 139:1069-1080.

59. Suttorp, N., U. Weber, T. Welsch, and C. Schudt. 1993. Role of phosphodiesterases in the regulation of endothelial permeability in vitro. J. Clin. Invest. 91:1421-1428.

60. Shatos, M., J. Doherty, D. Stump, E. Thompson, and D. Collen. 1990 Oxygen radicals generated using anoxia followed by reoxygenation reduce the synthesis of tissue-type plasminogen activator and plasminogen activator inhibitor-1 in human endothelial cell culture. J. Biol. Chem. 265:20443-20448.
61. Shatos, M., J. Doherty, and J. Hoak. 1991. Role of active oxygen species in the alteration of human vascular endothelial cell function: platelet adherence and prostacyclin release. Arterioscler. Thromb. 11:594-601.

62. Shatos, M., J. Doherty, T. Orfeo, J. Hoak, D. Collen, and D. Stump. 1992. Modulation of the fibrinolytic response by extracellularly generated oxygen radicals of cultured human vascular endothelium. J. Biol. Chem. 1:597-601.

63. Yoshida, N., D. Granger, D. Anderson, R. Rothlein, C. Lane, and P. Kvietys. 1992. Anoxia/reoxygenation-induce neutrophil adherence to cultured endothelial cells. Am. J. Physiol. 262:H1891-1898.

64. Shreeniwas, R., S. Koga, M. Karakurum, D. Pinsky, E. Kaiser, J. Brett, B. Wolitzky, C. Norton, J. Plocinski, W. Benjamin, et al. 1992. Hypoxia-mediated induction of endothelial cell interleukin- $1 \alpha$. An autocrine mechanism promoting expression of leukocyte adhesion molecules on the vessel surface. J. Clin. Invest. 90:2333-2339.

65. Pober, J., M. Slowik, L. DeLuca, and A. Ritchie. 1992. Elevated cAMP inhibits endothelial expression of ELAM-1 and VCAM-1 but not ICAM-1. FASEB (Fed. Am. Soc. Exp. Biol.) J. 6:3795.

66. Boxer, L., J. Allen, R. Baehner, and V. Amick. 1980. Diminished polymorphonuclear adherence. J. Clin. Invest. 66:268-274.

67. Simpson, P., J. Schelm, J. Smallwood, M. Clay, and T. Lindstrom. 1992. Inhibition of granulocyte cAMP-phosphodiesterase in vivo is not sufficient to protect the canine myocardium from reperfusion injury. J. Cardiovasc. Pharmacol. 19:987-955.

68. Rascon, A., S. Lindgren, L. Stavenow, P. Belfrage, K. Andersson, V. Manganiello, and E. Oegerman. 1992. Purification and properties of the cGMP inhibited phosphodiesterase from bovine aortic smooth muscle. Biochim. Biophys. Acta. 1134:149-156.

69. Marivet, M., J. Bourguignon, C. Lugnier, A. Mann, J. Stoclet, and W. Camille-Georges. 1989. Inhibition of adenosine-3',5'-monophosphate phosphodiesterase from vascular smooth muscle by rolipram analogues. J. Med. Chem. 32:1450-1457.

70. Engler, R., G. Schmid-Schonbein, and R. Pavalec. 1983. Leukocyte capillary plugging in myocardial ischemia and reperfusion in the dog. Am. J. Pathol. 111:98-111.

71. Engler, R., M. Dahlgren, D. Morris, M. Peterson, and G. Schmid-Schonbein. 1986. Role of leukocytes in response to acute myocardial ischemia and reflow in dogs. Am. J. Physiol. 251:H314-322.

72. Kloner, R. 1989. No reflow revisited. J. Am. Coll. Cardiol. 14:1814-1815.

73. Chen, C., H. Matsuda, Y. Sawa, M. Kaneko, N. Sakagoshi, N. Motonobu, T. Kuratani, A. Amemiya, and Y. Kawashima. 1991. Effect of a cyclic adenosine monophosphate phosphodiesterase inhibitor, DN-9693, on myocardial reperfusion injury. Ann. Thorac. Surg. 52:495-499.

74. Tanaka, K., F. Gotoh, Y. Fukuuchi, T. Amano, D. Uematsu, J. Kawamura, Y. Takemori, N. Itoh, K. Obara, and K. Muramatsu. 1989. Effects of a selective inhibitor of cyclic AMP phosphodiesterase on the pial microcirculation in feline cerebral ischemia. Stroke 20:668-673.

75. Mathew, R., H. Omar, P. Cherry, M. Gewitz, and M. Wolin. 1992. Role of CGMP mechanisms in response of rat pulmonary arteries to hypoxia. Am. J. Physiol. 263:H141-146.

76. Lowenstein, C., and S. Snyder. 1992. Nitric oxide, a novel biologic messenger. Cell. 70:705-707.

77. Ignarro, L., G. Ross, and J. Tillisch. 1991. Pharmacology of endotheliumderived nitric oxide and nitrovasodilators. West. J. Med. 154:51-62.

78. Pearl, J., H. Laks, D. Drinkwater, R. Byrns, L. Ignarro, and P. Chang. 1992. Loss of endothelial-dependent vasodilation and EDRF release following myocardial protection with University of Wisconsin (UW) solution. 86:I-763. (Abstr.)

79. Pinsky, D., S. Koga, M. Oz, A. Morales, R. Nowygrod, P. Cannon, and D. Stern. 1992. Failure of endogenous vasodilation contributes to cardiac graft failure following prolonged storage. Circulation. 86(4):I-763. (Abstr.)

80. Oz, M., D. Pinsky, S. Koga, V. Jeevanandam, M. Williams, S. Popilskis, C. Marboe, R. Nowygrod, D. Hsu, D. Stern, E. Rose, and R. Michler. 1992. Enhanced donor heart preservation with a novel cAMP based solution. Circulation. 86(4):I-840. (Abstr.) 